Supplementary Material

\title{
Electrical conductivity and spin crossover: a new achievement with a metal bis dithiolene complex
}

Christophe Faulmann, ${ }^{a}{ }^{*}$ Kane Jacob, ${ }^{a}$ Stéphane Dorbes, ${ }^{a}$ Stéphane Lampert, ${ }^{a}$ Isabelle Malfant, ${ }^{a}$ Marie-Liesse Doublet, ${ }^{b}$ Lydie Valade ${ }^{a}$ and José A. Real ${ }^{c}$

Table S1. Short intermolecular contacts in $\mathbf{1}$

Figure S1. Projection of the structure of 3 at $180 \mathrm{~K}$ along (dotted lines represent short intermolecular contacts)

Figure S2. Electronic band structure of 3 at $180 \mathrm{~K}$ calculated with the EHTB method for the five LUMO bands of the $\left[\mathrm{Ni}(\mathrm{dmit})_{2}\right]$ slab, with the projections in the reciprocal lattice $\mathrm{S}(-$ $1 / 2,1 / 2), \Gamma(0,0), \mathrm{X}(1 / 2,0), \mathrm{Y}(0,1 / 2)$ and $\mathrm{M}(1 / 2,1 / 2)$. 


\begin{tabular}{|c|c|c|c|c|c|c|}
\hline \multirow[t]{2}{*}{ Entities } & \multirow[t]{2}{*}{ Atom1 } & \multirow[t]{2}{*}{ Atom2 } & \multirow{2}{*}{\multicolumn{2}{|c|}{$\begin{array}{l}\text { Symmetry operation to be applied } \\
\text { on atom } 1 \quad \text { on atom } 2\end{array}$}} & \multicolumn{2}{|c|}{ Bond length } \\
\hline & & & & & at $293 \mathrm{~K}$ & at $180 \mathrm{~K}$ \\
\hline $\multirow{21}{*}{\mathrm{Ni}}-\{\mathrm{Fe}\}$ & S205 & $\mathrm{H} 2$ & $-x, 1-y,-z$ & $\mathrm{x}, 1+\mathrm{y}, \mathrm{z}$ & 2.772 & 2.743 \\
\hline & S205 & $\mathrm{H} 4$ & $-\mathrm{x}, 1-\mathrm{y},-\mathrm{z}$ & $\mathrm{x}, 1+\mathrm{y}, \mathrm{z}$ & 2.636 & 2.596 \\
\hline & C203 & H52A & $-\mathrm{x}, 1-\mathrm{y},-\mathrm{z}$ & $\mathrm{x}, 1+\mathrm{y}, \mathrm{z}$ & 2.874 & 2.801 \\
\hline & S205 & $\mathrm{H} 2$ & $\mathrm{x}, \mathrm{y}, \mathrm{z}$ & $-x,-y,-z$ & 2.772 & 2.743 \\
\hline & S205 & $\mathrm{H} 4$ & $\mathrm{x}, \mathrm{y}, \mathrm{z}$ & $-\mathrm{x},-\mathrm{y},-\mathrm{z}$ & 2.636 & 2.596 \\
\hline & C203 & H52A & $\mathrm{x}, \mathrm{y}, \mathrm{z}$ & $-\mathrm{x},-\mathrm{y},-\mathrm{z}$ & 2.874 & 2.801 \\
\hline & $\mathrm{H} 41 \mathrm{C}$ & S105 & $\mathrm{x}, \mathrm{y}, \mathrm{z}$ & $-\mathrm{x},-\mathrm{y},-\mathrm{z}$ & 2.982 & 2.995 \\
\hline & H52B & S103 & $\mathrm{x}, \mathrm{y}, \mathrm{z}$ & $1-x,-y,-z$ & 2.927 & 2.873 \\
\hline & $\mathrm{H} 2$ & S205 & $\mathrm{x}, \mathrm{y}, \mathrm{z}$ & $-\mathrm{x},-\mathrm{y},-\mathrm{z}$ & 2.772 & 2.743 \\
\hline & $\mathrm{H} 4$ & S205 & $\mathrm{x}, \mathrm{y}, \mathrm{z}$ & $-\mathrm{x},-\mathrm{y},-\mathrm{z}$ & 2.636 & 2.596 \\
\hline & H52A & $\mathrm{C} 203$ & $\mathrm{x}, \mathrm{y}, \mathrm{z}$ & $-\mathrm{x},-\mathrm{y},-\mathrm{z}$ & 2.874 & 2.801 \\
\hline & S103 & H52B & $1-x, 1-y,-z$ & $\mathrm{x}, 1+\mathrm{y}, \mathrm{z}$ & 2.927 & 2.873 \\
\hline & S105 & $\mathrm{H} 41 \mathrm{C}$ & $1-x, 1-y,-z$ & $1+x, 1+y, z$ & 2.982 & 2.995 \\
\hline & S105 & $\mathrm{H} 41 \mathrm{C}$ & $\mathrm{x}, \mathrm{y}, \mathrm{z}$ & $-\mathrm{x},-\mathrm{y},-\mathrm{z}$ & 2.982 & 2.995 \\
\hline & S103 & H52B & $\mathrm{x}, \mathrm{y}, \mathrm{z}$ & $1-x,-y,-z$ & 2.927 & 2.873 \\
\hline & S101 & H49A & $\mathrm{x}, \mathrm{y}, \mathrm{z}$ & $1+x, y, z$ & & 2.969 \\
\hline & S203 & H41B & $\mathrm{x}, \mathrm{y}, \mathrm{z}$ & $1+\mathrm{x}, \mathrm{y}, \mathrm{z}$ & & 2.975 \\
\hline & S203 & H41B & $-\mathrm{x}, 1-\mathrm{y},-\mathrm{z}$ & $-1-x, 1-y,-z$ & & 2.975 \\
\hline & H49A & S101 & $\mathrm{x}, \mathrm{y}, \mathrm{z}$ & $-1+x, y, z$ & & 2.969 \\
\hline & H41B & S203 & $\mathrm{x}, \mathrm{y}, \mathrm{z}$ & $-1+x, y, z$ & & 2.975 \\
\hline & S101 & H49A & $1-x, 1-y,-z$ & $-\mathrm{x}, 1-\mathrm{y},-\mathrm{z}$ & & 2.969 \\
\hline $\multirow{14}{*}{\mathrm{Fe}}-\{\mathrm{Fe}\}$ & $\mathrm{C} 43$ & H33 & $\mathrm{x}, \mathrm{y}, \mathrm{z}$ & $-1+x, y, z$ & 2.751 & 2.713 \\
\hline & H33 & $\mathrm{C} 43$ & $\mathrm{x}, \mathrm{y}, \mathrm{z}$ & $1+\mathrm{x}, \mathrm{y}, \mathrm{z}$ & 2.751 & 2.713 \\
\hline & $\mathrm{H} 50 \mathrm{~A}$ & C52 & $\mathrm{x}, \mathrm{y}, \mathrm{z}$ & $-\mathrm{x},-\mathrm{y},-\mathrm{z}$ & 2.772 & 2.696 \\
\hline & C52 & $\mathrm{H} 50 \mathrm{~A}$ & $\mathrm{x}, \mathrm{y}, \mathrm{z}$ & $-\mathrm{x},-\mathrm{y},-\mathrm{z}$ & 2.772 & 2.696 \\
\hline & $\mathrm{C} 32$ & $\mathrm{H} 44$ & $\mathrm{x}, \mathrm{y}, \mathrm{z}$ & $-\mathrm{x}, 1-\mathrm{y}, 1-\mathrm{z}$ & 2.861 & 2.814 \\
\hline & $\mathrm{H} 44$ & $\mathrm{C} 32$ & $\mathrm{x}, \mathrm{y}, \mathrm{z}$ & $-\mathrm{x}, 1-\mathrm{y}, 1-\mathrm{z}$ & 2.861 & 2.814 \\
\hline & $\mathrm{H} 44$ & $\mathrm{O} 32$ & $\mathrm{x}, \mathrm{y}, \mathrm{z}$ & $-x, 1-y, 1-z$ & 2.672 & 2.626 \\
\hline & $\mathrm{O} 32$ & $\mathrm{H} 44$ & $\mathrm{x}, \mathrm{y}, \mathrm{z}$ & $-\mathrm{x}, 1-\mathrm{y}, 1-\mathrm{z}$ & 2.672 & 2.626 \\
\hline & $\mathrm{C} 45$ & $\mathrm{C} 43$ & $\mathrm{x}, \mathrm{y}, \mathrm{z}$ & $-\mathrm{x}, 1-\mathrm{y}, 1-\mathrm{z}$ & & 3.389 \\
\hline & C53 & $\mathrm{H} 54 \mathrm{C}$ & $\mathrm{x}, \mathrm{y}, \mathrm{z}$ & $-\mathrm{x}, 1-\mathrm{y}, 1-\mathrm{z}$ & & 2.867 \\
\hline & $\mathrm{H} 54 \mathrm{C}$ & C53 & $\mathrm{x}, \mathrm{y}, \mathrm{z}$ & $-x, 1-y, 1-z$ & & 2.867 \\
\hline & $\mathrm{C} 42$ & $\mathrm{C} 44$ & $\mathrm{x}, \mathrm{y}, \mathrm{z}$ & $-\mathrm{x}, 1-\mathrm{y}, 1-\mathrm{z}$ & & 3.343 \\
\hline & $\mathrm{C} 43$ & $\mathrm{C} 45$ & $\mathrm{x}, \mathrm{y}, \mathrm{z}$ & $-x, 1-y, 1-z$ & & 3.389 \\
\hline & $\mathrm{C} 44$ & $\mathrm{C} 42$ & $\mathrm{x}, \mathrm{y}, \mathrm{z}$ & $-x, 1-y, 1-z$ & & 3.343 \\
\hline $\multirow{9}{*}{\mathrm{Fe}}-\{$ solv. $\}$ & $\mathrm{O} 2$ & H90 & $\mathrm{x}, \mathrm{y}, \mathrm{z}$ & $\mathrm{x}, \mathrm{y}, \mathrm{z}$ & 2.701 & 2.483 \\
\hline & $\mathrm{C} 37$ & O90 & $\mathrm{x}, \mathrm{y}, \mathrm{z}$ & $\mathrm{x}, \mathrm{y}, \mathrm{z}$ & 3.021 & 3.011 \\
\hline & C54 & H90C & $\mathrm{x}, \mathrm{y}, \mathrm{z}$ & $\mathrm{x}, \mathrm{y}, \mathrm{z}$ & 2.718 & 2.726 \\
\hline & H54B & $\mathrm{H} 90 \mathrm{C}$ & $\mathrm{x}, \mathrm{y}, \mathrm{z}$ & $\mathrm{x}, \mathrm{y}, \mathrm{z}$ & 2.357 & 2.350 \\
\hline & O43 & O90 & $\mathrm{x}, \mathrm{y}, \mathrm{z}$ & $\mathrm{x}, \mathrm{y}, \mathrm{z}$ & 2.960 & 2.934 \\
\hline & $\mathrm{O} 43$ & $\mathrm{H} 90$ & $\mathrm{x}, \mathrm{y}, \mathrm{z}$ & $\mathrm{x}, \mathrm{y}, \mathrm{z}$ & 2.214 & 2.206 \\
\hline & $\mathrm{O} 43$ & H90C & $\mathrm{x}, \mathrm{y}, \mathrm{z}$ & $\mathrm{x}, \mathrm{y}, \mathrm{z}$ & 2.682 & 2.669 \\
\hline & O90 & $\mathrm{H} 37$ & $\mathrm{x}, \mathrm{y}, \mathrm{z}$ & $-x,-y, 1-z$ & & 2.630 \\
\hline & H37 & O90 & $\mathrm{x}, \mathrm{y}, \mathrm{z}$ & $-x,-y, 1-z$ & & 2.630 \\
\hline $\multirow{3}{*}{\mathrm{Ni}}-\{\mathrm{Ni}\}$ & S101 & $\mathrm{C} 203$ & $\mathrm{x}, \mathrm{y}, \mathrm{z}$ & $\mathrm{x}, \mathrm{y}, \mathrm{z}$ & 3.479 & 3.466 \\
\hline & S101 & $\mathrm{C} 203$ & $1-x, 1-y,-z$ & $1-x, 1-y,-z$ & 3.479 & 3.466 \\
\hline & C203 & S101 & $-\mathrm{x}, 1-\mathrm{y},-\mathrm{z}$ & $-\mathrm{x}, 1-\mathrm{y},-\mathrm{z}$ & 3.479 & 3.466 \\
\hline
\end{tabular}

- $\{\mathrm{Ni}\},\{\mathrm{Fe}\}$ and $\{$ solv. $\}$ stand for $\left[\mathrm{Ni}(\mathrm{dmit})_{2}\right],\left[\mathrm{Fe}(3-\mathrm{OMe}-\mathrm{salEen})_{2}\right]$ and methanol, respectively

Table S1. Short intermolecular contacts in $\mathbf{1}$ 


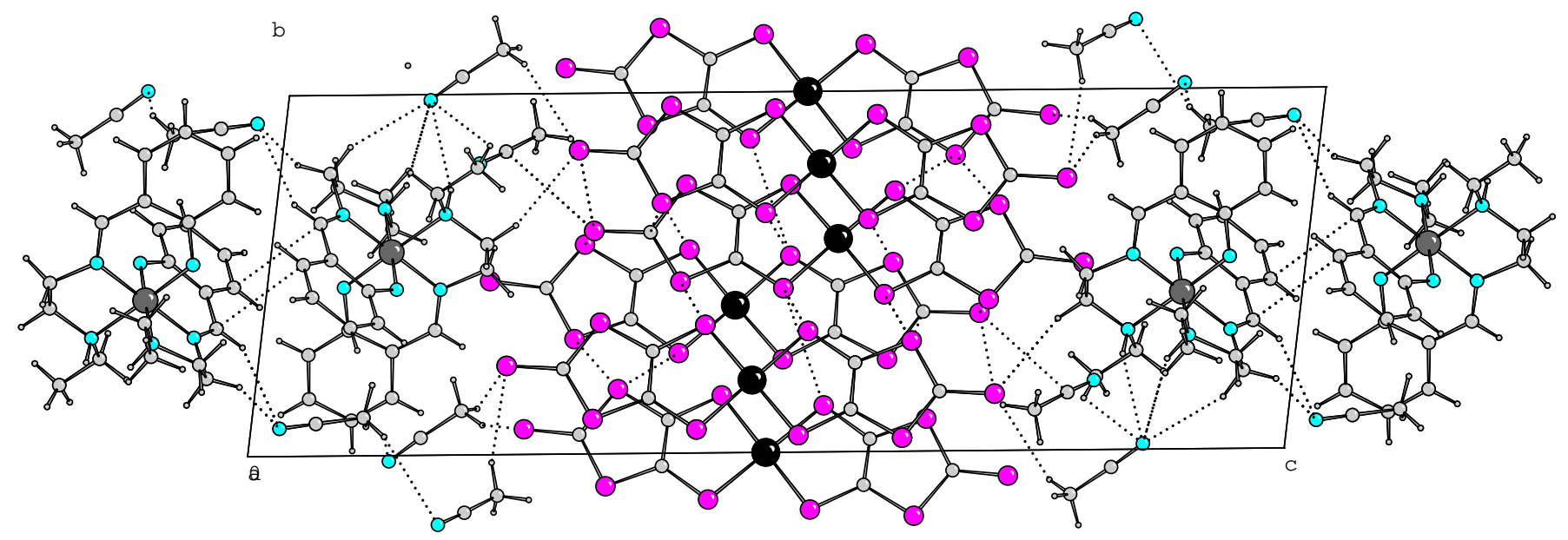

Figure S1. Projection of the structure of $\mathbf{3}$ at $180 \mathrm{~K}$ along (dotted lines represent short intermolecular contacts)

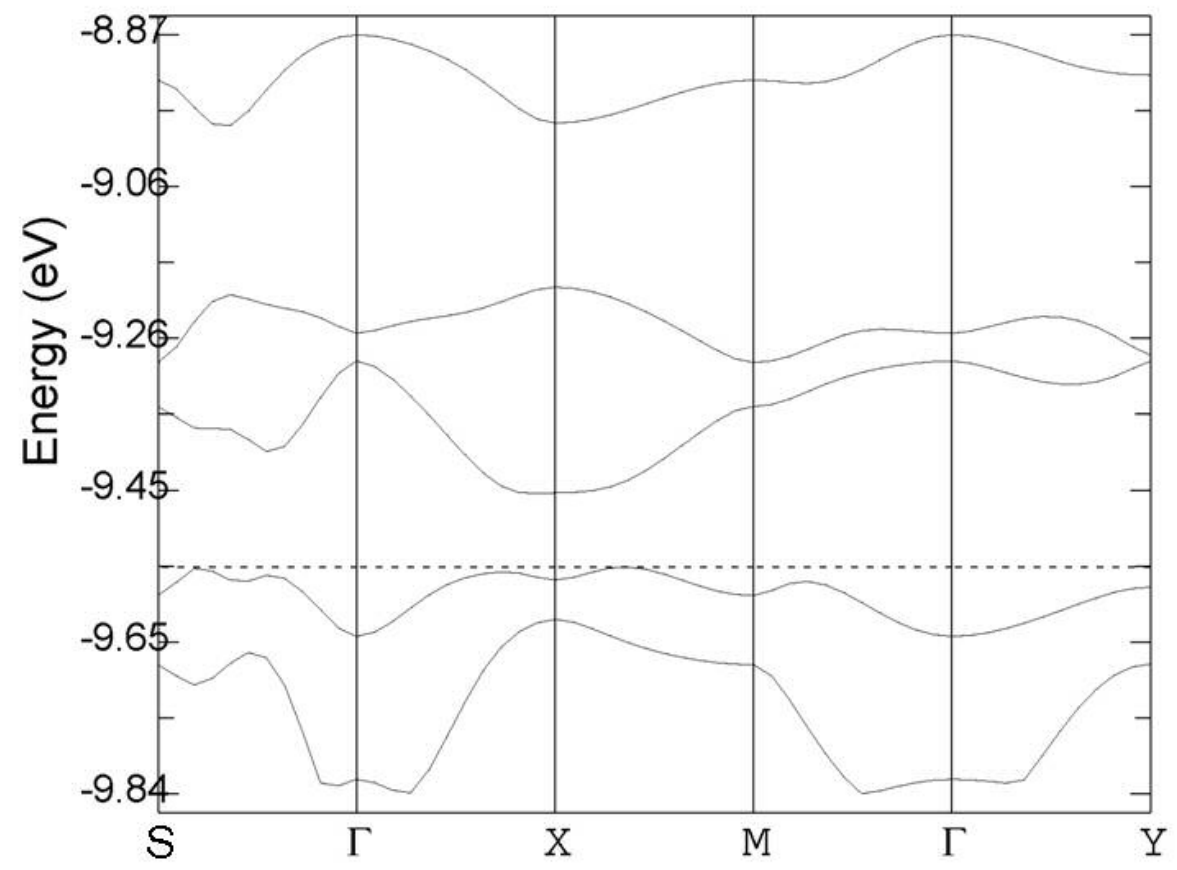

Figure S2. Electronic band structure of 3 at $180 \mathrm{~K}$ calculated with the EHTB method for the five LUMO bands of the $\left[\mathrm{Ni}(\mathrm{dmit})_{2}\right]$ slab, with the projections in the reciprocal lattice $\mathrm{S}(-$ $1 / 2,1 / 2), \Gamma(0,0), \mathrm{X}(1 / 2,0), \mathrm{Y}(0,1 / 2)$ and $\mathrm{M}(1 / 2,1 / 2)$. 